\title{
Formation des médecins et infirmières à l'utilisation des opiacés par un jeu sérieux
}

\section{Training of Doctors and Nurses in the Use of Opiates by a Serious Game}

\author{
C. Bollondi Pauly $\cdot$ S. Kupferschmid $\cdot$ P. Tairraz $\cdot$ C. Samer $\cdot$ B. Rehberg-Klug \\ Reçu le 07 janvier 2019; accepté le 20 janvier 2019 \\ (C) Lavoisier SAS 2019
}

\begin{abstract}
Résumé Malgré les formations existantes, de nouvelles stratégies pédagogiques sont nécessaires afin d'améliorer les compétences des professionnels concernant la prescription et la dispensation des opiacés. La construction d'un jeu sérieux permet d'expérimenter dans un cadre sécurisant des habilités cliniques. Les modalités de conception du contenu théorique, des aspects interactionnels et ludiques développés sont présentés. Alors que la satisfaction des participants est élevée, les résultats concernant la participation des professionnels montrent les enjeux relatifs au déploiement.
\end{abstract}

Mots clés Jeu sérieux - Formation médicale et infirmière Utilisation des opioïdes

Abstract Despite all educative efforts, medical caregivers
continue to have only limited theoretical and practical know-
ledge of opioid therapy. New resources are necessary to help

C. Bollondi Pauly $(\varangle) \cdot$ S. Kupferschmid $(\square)$

Direction adjointe des soins, hôpitaux universitaires de Genève,

4, rue Gabrielle-Perret-Gentil, CH-1205 Genève, Suisse

e-mail : catherine.bollondi@hcuge.ch,

sarah.kupferschmid@hcuge.ch

P. Tairraz $(\bowtie)$

Direction des ressources humaines,

centre de formation et compétences,

hôpitaux universitaires de Genève,

rue Alcide-Jentzer, CH-1211 Genève 14, Suisse

e-mail : paul.Tairraz@hcuge.ch

C. Samer $(\bowtie)$

Service de pharmacologie et toxicologie clinique,

département de médecine aiguë,

hôpitaux universitaires de Genève,

4, rue Gabrielle-Perret-Gentil, CH-1205 Genève, Suisse

e-mail : caroline.samer@hcuge.ch

B. Rehberg-Klug $(\square)$

Service d'anesthésiologie, département de médecine aiguë,

hôpitaux universitaires de Genève, 4, rue Gabrielle-Perret-Gentil,

CH-1205 Genève, Suisse

e-mail : benno.Rehberg-klug@hcuge.ch improve prescription and distribution of opioids. The development of a "serious game" may provide training in a secure environment. Here the development of the content and the possibilities of interprofessional interaction of such an e-learning are described. While satisfaction of participants was shown to be high, the rates of participation show the difficulties of implementing this type of training.

Keywords Serious game - Medical and nurse education · Opioids use

\section{À vous de jouer !}

À vous de jouer!

http://digifor.s3-eu-west-3.amazonaws.com/opiaces_hug/ story.html

L'enquête de satisfaction menée chaque année auprès des patients hospitalisés dans un hôpital universitaire de Genève montre que $58 \%$ des patients présentent des douleurs pendant leur séjour hospitalier. Quatre-vingt-dix pour cent d'entre eux ressentent une douleur modérée à forte. Une donnée importante de l'enquête de satisfaction de l'institution nous révèle que près d'un quart des patients hospitalisés estiment que le personnel soignant n'a pas fait tout son possible pour soulager la douleur. Une étude qui avait été réalisée au sein de notre institution avait mis en évidence la réticence des infirmières à administrer des réserves d'opiacés malgré leur prescription, et cela par crainte d'effets indésirables, alors que le patient manifeste une douleur d'intensité modérée à forte [1]. L'utilisation des antalgiques, notamment des opiacés, nécessite une formation continue et des connaissances pharmacologiques et cliniques régulièrement mises à jour $[2,3]$. Les données de la littérature indiquent que les erreurs les plus communes des médecins prescripteurs concernent la dose prescrite et les ajustements thérapeutiques, ainsi que l'absence de traitement de prévention des effets indésirables et d'antalgiques pour les gestes iatrogènes douloureux [4,5]. 
L'OMS souligne par ailleurs le rôle primordial que les patients et les usagers peuvent jouer pour améliorer la sécurité et la qualité des soins dans le monde [6]. Le partenariat patient-soignant partenaires, tel que celui-ci est promu par l'hôpital universitaire de Genève, s'exprime par un changement de mode relationnel en faveur d'une collaboration accrue entre les patients, leurs aidants et les professionnels. Le patient partenaire est celui qui construit avec les professionnels une recherche de solutions adaptées à des problématiques concernant sa santé et son projet de soins ou à des questions de stratégie de santé [7]. Le nombre de médecins et d'infirmières au sein d'un hôpital universitaire ainsi que la rotation du personnel exigent enfin la mise en place des stratégies de formation adaptées.

Ces éléments montrent que, malgré les actions développées autour de cette thématique ainsi que les formations dispensées, la douleur et sa prise en charge dans une relation de partenariat restent un défi qu'il convient d'essayer de surmonter. La formation doit s'envisager dans la durée et être facilement accessible.

Dans le domaine de l'enseignement, les jeux sérieux apparaissent comme des outils pédagogiques innovants pour acquérir des compétences, notamment dans le domaine de la santé. Il s'agit d'applications logicielles utilisant les principes et la mécanique du ludique pour atteindre des objectifs sérieux tels que la formation ou la motivation à suivre un traitement médical par exemple [8], y compris pour la douleur [9].

Ils permettent de diminuer les coûts opérationnels en permettant un accès large à la formation [10]. Le principal intérêt du jeu sérieux réside dans sa proximité avec une situation professionnelle réelle, permettant au soignant d'expérimenter, dans un cadre sécurisant, des habilités cliniques qu'il développe. Le transfert des acquis en est favorisé $[11,12]$. Ce support pédagogique peut être diffusé largement via une plateforme informatique permettant l'accès des professionnels à partir de tout poste informatique.

\section{Objectifs}

L'amélioration de la qualité et de la sécurité de la prescription de traitements antalgiques opiacés est le premier objectif visé par la démarche de mise en place d'un jeu sérieux sur l'apprentissage de la gestion des opiacés, notamment lors de la maladie oncologique. Les autres buts du jeu sérieux sont d'accentuer le partenariat ainsi que la collaboration interprofessionnelle et d'améliorer au bout du compte l'information et le traitement de prise en charge symptomatique du patient. Cette démarche permet de compléter, de façon innovante et ludique, les enseignements existants pour améliorer les connaissances et compétences cliniques (théoriques et pratiques) des médecins et des infirmiers dans la prise en charge de patients souffrant de douleurs cancéreuses et non cancéreuses.

\section{Construction du jeu}

Un groupe d'experts médicaux et infirmiers dans le domaine de l'antalgie a collaboré avec un expert des technologies de l'information et de la pédagogie pendant dix mois pour concevoir le jeu sérieux.

Un scénario spécifique pour les médecins et un autre spécifique pour les infirmiers ont été conçus autour d'une même situation d'un patient hospitalisé pour une acutisation de ses douleurs aiguës cancéreuses du pancréas.

Le financement de la conception et de la réalisation du graphisme par une société privée experte dans la création digitale a été possible grâce à la fondation privée des hôpitaux universitaires de Genève qui soutient différents projets de l'institution.

Le contenu a été conçu par les experts de l'institution, permettant à celle-ci d'être indépendante dans l'ajustement du contenu.

Les connaissances transmises lors de la formation sont déclinées autour des trois thématiques suivantes :

\section{Connaissances théoriques}

L'évaluation de la douleur, de l'efficacité et de la tolérance du traitement a été travaillée en tenant compte des facteurs de risque ainsi qu'en intégrant l'observance médicamenteuse.

La prescription, l'administration et la surveillance des traitements par opiacés sont déclinées avec un rappel des principes pharmacologiques (pharmacocinétiques et pharmacodynamiques) pour une prescription rationnelle et sûre (molécules, galéniques, rotation d'opiacés et doses équianalgésiques).

La prévention ainsi que l'identification des effets indésirables et leurs traitements sont présentés. Les sources de variabilité interindividuelle dans la réponse aux opiacés sont décrites (par exemple variabilité pharmacogénétique) ainsi que les précautions de prescription à prendre lors d'une atteinte rénale ou hépatique, d'interactions médicamenteuses pharmacocinétiques et pharmacodynamiques.

Les documents ressources d'aide à l'évaluation, d'aide à la prescription ou à l'information du patient sont présentés comme support tout au long des scénarios afin de permettre leur appropriation par les professionnels et rendre ainsi ces derniers plus autonomes dans leur exercice.

\section{Collaboration interprofessionnelle}

L'échange d'informations entre professionnels, concernant l'évaluation de l'état clinique du patient, sa réponse au 
traitement, la décision thérapeutique, est également travaillé à plusieurs reprises dans les scénarios au travers des interactions entre les professionnels.

\section{Partenariat patient-professionnel}

Les questions des patients, leur perception ainsi que leurs préoccupations ont été utilisées afin d'être au plus près des situations vécues par les patients. Douze entretiens semistructurés avec des patients hospitalisés en orthopédie, en médecine interne générale, en médecine de réhabilitation et en oncologie ont été menés. Ils ont permis de recueillir l'expérience de l'évaluation de la douleur et de sa prise en charge thérapeutique lorsque les traitements par morphine ou ses dérivés étaient employés.

L'implication des patients dans ce projet correspond au niveau de partenariat selon le modèle développé dans l'institution : les patients expriment et délibèrent sur leur prise en charge de la douleur et collaborent ainsi indirectement à la construction d'une formation pour les professionnels de la santé. Les thèmes identifiés comme étant problématiques au cours des expériences des patients ont nourri les échanges patients-professionnels dans les différents scénarios élaborés.

Les dialogues ont été enrichis en reprenant certaines phrases des patients mot à mot. Les principes de communication dans le domaine de la santé [13] ont contribué à l'élaboration du dialogue entre le patient et le professionnel.

Les entretiens ont montré l'intérêt exprimé par plusieurs patients d'être informés de façon détaillée sur les traitements opiacés. Ainsi, ils souhaitent pouvoir comprendre l'usage des opioïdes, en particulier lorsqu'ils doivent être pris de façon régulière, mais aussi du principe des réserves d'opioïdes. Cette compréhension améliorée leur permet de mieux collaborer ensuite avec les équipes soignantes en signalant plus rapidement la résurgence et/ou l'apparition de la douleur, plutôt que d'essayer de résister à celle-ci dans le but de limiter le nombre de doses supplémentaires. La continuité du traitement dans le parcours de soins et le retour au domicile sont apparus également comme un élément pouvant poser problème du point de vue de l'expérience d'un patient, l'arrêt brutal des antalgiques ayant pu entraîner une nouvelle hospitalisation pour celui-ci. Un patient a par ailleurs souligné l'importance du dialogue dans la mise en place et l'ajustement d'un traitement; il a transmis par exemple l'importance de respecter ses perceptions (effets perçus du traitement), alors que son témoignage avait été qualifié de non pertinent avec les effets connus du traitement par un professionnel. D'autres craintes exprimées par les patients doivent être prises en compte comme celles concernant principalement le risque de dépendance, tout comme celles rapportées de proches du « risque de s'habituer ». Les données recueillies auprès des patients ont confirmé les perceptions des experts.
Outre les aspects théoriques dans une situation emblématique, la mise en forme permet de rendre l'outil immersif, interactionnel et ludique.

\section{Réalisme}

Afin de rendre l'environnement et les activités proches de la réalité des professionnels, le graphisme représente les nouveaux locaux de l'institution. Dans le même objectif, une maquette du dossier informatique « patient intégré » a été créée afin que le professionnel puisse en cours de jeu consulter les données du patient (laboratoire, imagerie, notes de suite, graphique des signes vitaux) comme il le fait dans la pratique quotidienne. Des sons recréant l'ambiance ont été insérés tels que celui d'une sonnette et d'un téléphone à différents moments des scénarios imaginés.

Des médecins ou infirmiers cadres cliniques interviennent dans le déroulement de la prise en charge afin de questionner ou d'apporter de l'information comme c'est le cas lors des visites médicales ou infirmières dans un hôpital universitaire.

\section{Aspects interactionnels et ludiques}

Le participant est « challengé » à tester ses connaissances au cours de la simulation en termes de calcul de doses ou de choix de formulation dans les dialogues avec le patient. Il doit mettre en relation des éléments constitutifs d'une anamnèse douleur. La formation se termine avec un quiz afin de reprendre les messages clés. Le graphisme du quiz évoque l'environnement et les conditions de jeux télévisés populaires. Le participant prend connaissance de son score de réussite qu'il peut améliorer en répétant le quiz. Les activités ont été paramétrées afin de rendre le participant actif selon un rythme déterminé.

\section{Déploiement}

Après des tests des prototypes itératifs avec des utilisateurs du public cible [14], le jeu sérieux a été déployé en avril 2018 auprès d'environ 6000 professionnels médecins et infirmiers après une information des cadres de l'institution. L'information a été relayée par les cellules douleur des différents services du réseau transversal douleur de l'institution, appuyée par une communication sur le site intranet de l'institution. La réalisation du jeu est prévue de façon individuelle au moment choisi par le participant avec le soutien des responsables d'équipe. Dans certains services, le jeu sérieux est effectué lors de moments de formation. Il est alors utilisé en petits groupes, suscitant la discussion autour des questions soulevées. 


\section{Participation et satisfaction}

Après neuf mois de déploiement, le taux de participation général des professionnels médico-infirmiers est de $24 \%$ $(n=6137): 33 \%$ des infirmières ont réalisé le jeu sérieux, et près de $10 \%$ des médecins. La participation varie selon les secteurs et prédomine dans les secteurs d'oncologie (37\%), de médecine générale ( $36 \%$ ), de réhabilitation et de médecine palliative $(35 \%)$, et de chirurgie $(33 \%)$. Le département de psychiatrie atteint une participation de $11 \%$. Après un engouement au lancement du jeu durant les trois premiers mois (environ 300 participants par mois), les mois suivants comptent une moyenne régulière de 100 réalisations.

À la fin de la réalisation du jeu sérieux, une évaluation est proposée aux participants. Durant la période décrite, 524 éva- luations ont été renseignées (36 \%). L'enquête montre une forte satisfaction des participants tant au niveau du concept pédagogique (bien à très bien : $99 \%$ ) (Fig. 1) et des activités proposées (bien à très bien : $98 \%$ ) (Fig. 2) que de la concordance du contenu avec les besoins (bien à très bien : $97 \%$ ) (Fig. 3) et avec les objectifs de formation (bien à très bien : $99 \%$ ) (Fig. 4). L'ergonomie et la technologie reçoivent une satisfaction au-delà de $90 \%$ également.

\section{Discussion}

La force du jeu est un contenu que les professionnels reconnaissent comme correspondant à leurs besoins dans un environnement familier. La concordance des contenus avec les

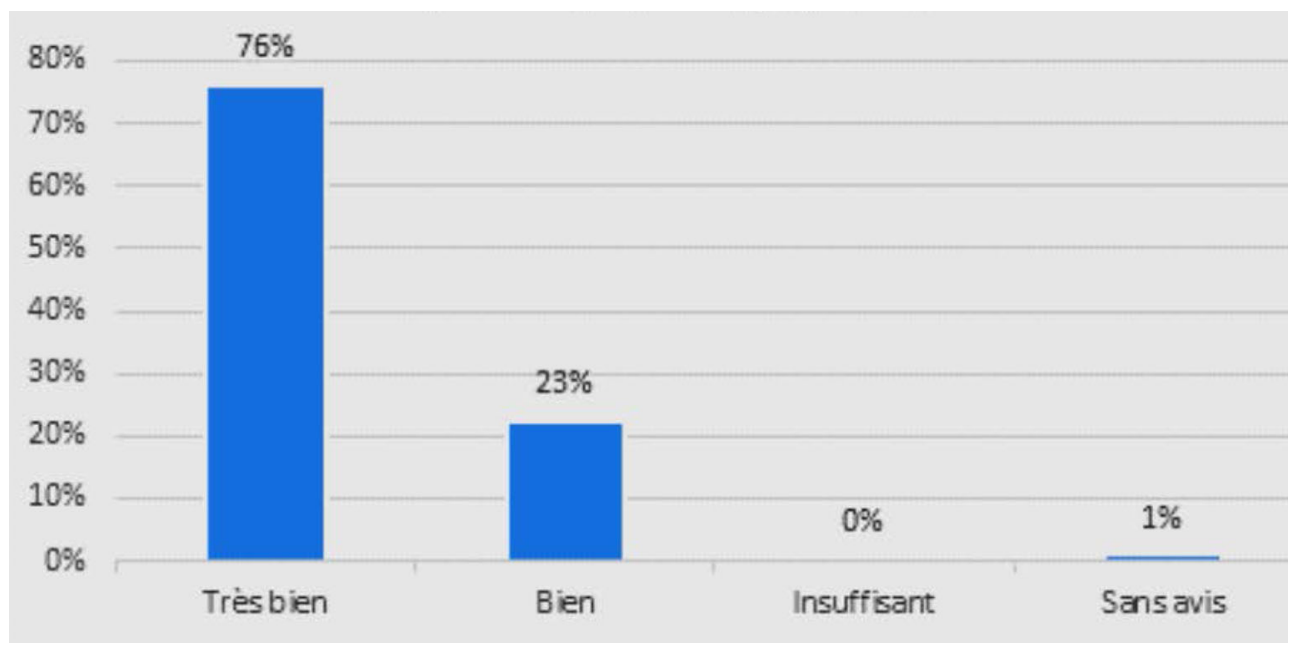

Fig. 1 Concept pédagogique

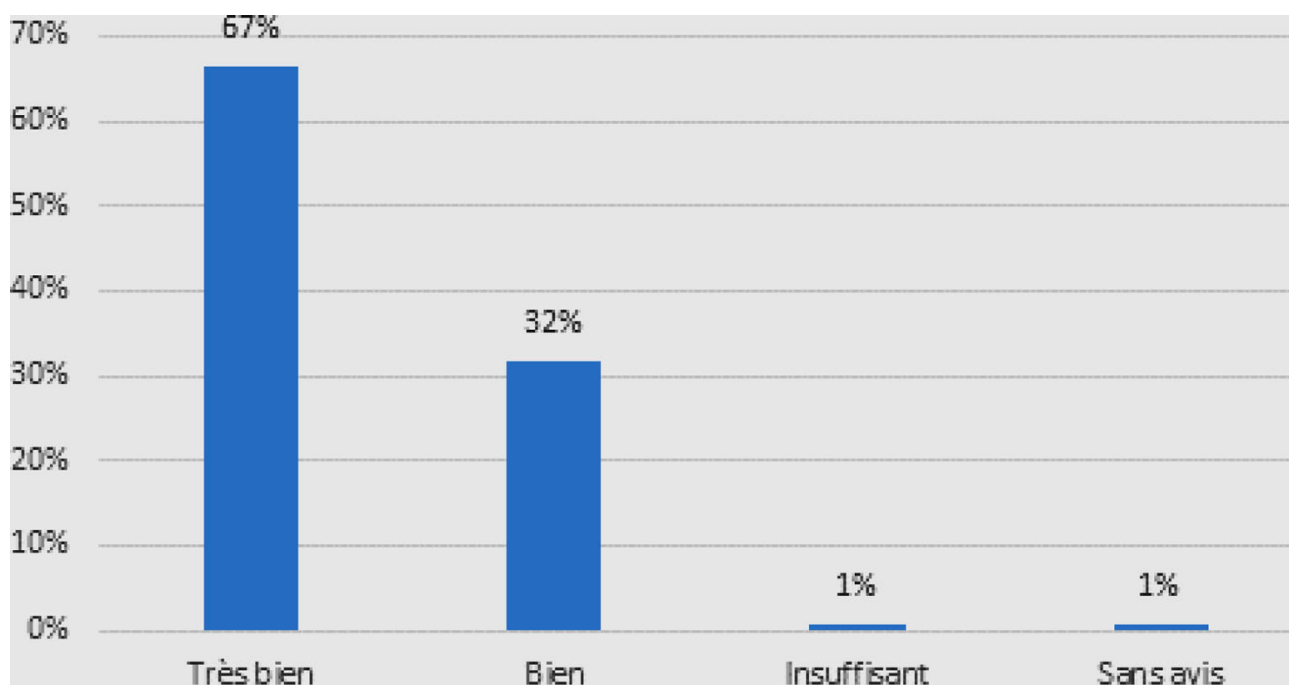

Fig. 2 Les activités 


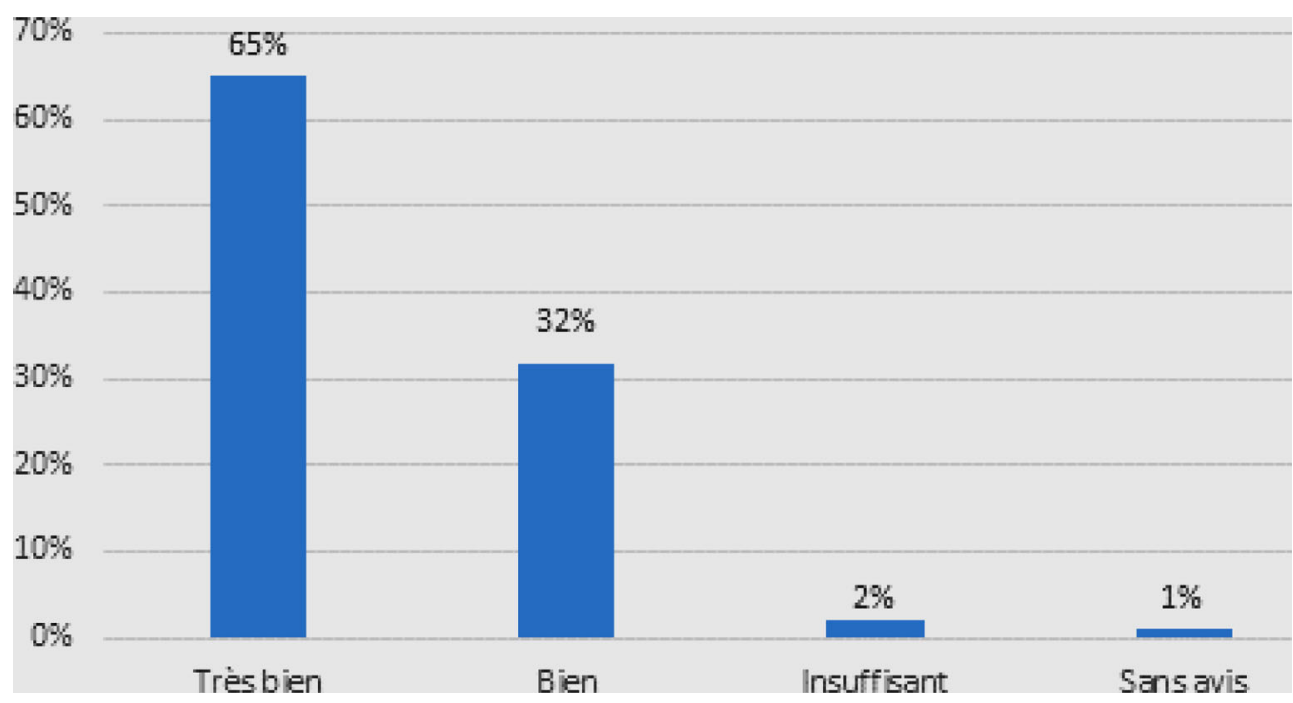

Fig. 3 Le contenu répond à vos besoins

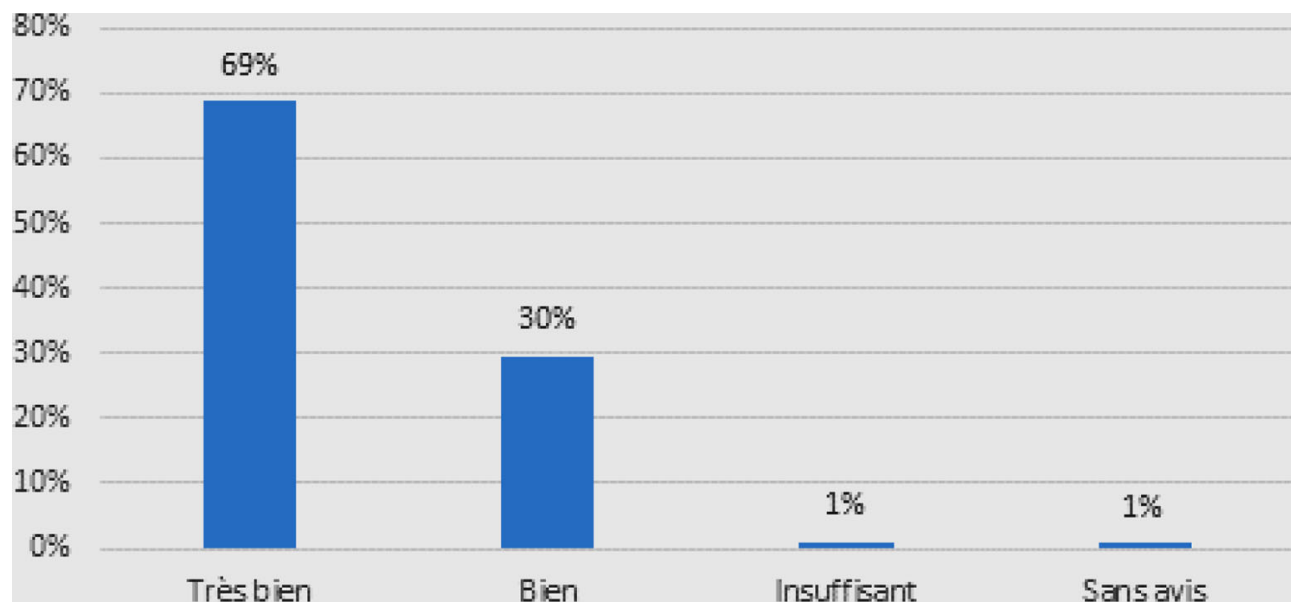

Fig. 4 Concordance et qualité des contenus avec les objectifs de formation

objectifs de la formation est également bien évaluée par les participants. Ces résultats permettent de considérer que le contenu couvre adéquatement les dimensions de l'activité qu'il vise à éduquer. Ce point est un des critères de validité des jeux d'éducation de professionnels médicaux [15]. Les résultats de cette enquête ne nous permettent cependant pas d'évaluer les autres critères de validité tels que la concordance des résultats de l'enseignement par le jeu en comparaison avec une méthode classique d'enseignement concernant le contenu théorique. Une évaluation des connaissances acquises au décours du jeu devrait être mesurée. Ainsi, les résultats permettraient d'évaluer également les différences inhérentes au degré de compétence des participants novices et experts, démontrant ainsi le caractère significatif des compétences paramétrées par le jeu.
Le versant ludique du dispositif est important. En effet, si la simulation ne peut à elle seule permettre de qualifier le dispositif de jeu, l'usage d'un patient virtuel, associé à des éléments de compétition sous forme de score que l'on peut améliorer ou de récompenses, peut être considéré comme intégrant les principes du jeu sérieux [16]. Ainsi, ce dispositif correspond aux critères du jeu sérieux, mais pourrait sans doute être amélioré en développant un caractère compétitif. Le participant par exemple pourrait être « challengé » à franchir différentes étapes avec des niveaux de connaissances progressifs. En effet, le niveau de challenge proposé est directement corrélé à l'engagement du professionnel avec des effets positifs sur l'apprentissage [17].

Différents critères du dispositif de simulation favorisent l'acquisition des connaissances : les effets sonores ou visuels 
permettent de susciter et de maintenir un niveau d'alerte qui contribue à préserver l'attention du participant [18]. Les informations émanant de l'environnement dans le jeu favorisent également l'apprentissage, le participant devant chercher lui-même des indices comme c'est le cas grâce à la maquette informatique du dossier patient intégré familier au soignant dans l'institution.

Les résultats élevés de satisfaction montrent un certain engouement des participants pour ce mode pédagogique. Il est reconnu par plusieurs auteurs que, si les jeux sérieux dans le milieu médical offrent des résultats similaires à d'autres méthodes [19], ils peuvent fournir un soutien supplémentaire pour engager les participants et améliorer leur satisfaction [20]. L'intégration du dispositif de formation par simulation au sein de programmes de formation optimise l'impact sur le transfert des connaissances et ainsi la prévention des erreurs médicales. Ainsi, les dispositions prises par le réseau douleur de l'institution consistant à insérer le jeu sérieux dans les programmes existants en juxtaposition avec l'intervention d'experts favorisent sa plus-value.

\section{Perspectives}

Après la phase de déploiement, il apparaît que les enjeux concernent maintenant la pérennisation de cette formation au sein de l'institution. Le soutien opérationnel des cadres pour informer, encourager, voire recommander cette formation auprès des collaborateurs apparaît primordial, mais aussi afin de permettre à ceux-ci d'effectuer le jeu sur leur temps de travail.

La difficulté de toucher les médecins de l'institution est récurrente à d'autres types de formations. Ces derniers ayant un curriculum de formation académique propre, leur disponibilité à d'autres démarches de formation est faible. Une reconnaissance de cette formation courte interactive est en cours auprès de sociétés de différentes disciplines médicales afin de favoriser l'intégration de la formation dans le cursus académique des médecins.

L'équipe projet construit un questionnaire pour évaluer les acquis au niveau des connaissances et des compétences des médecins et des infirmières afin d'évaluer la plusvalue de ce mode pédagogique. Le questionnaire sera administré aux participants avant, après et à distance de la formation.

La perspective d'adapter le jeu à une situation de pédiatrie est à l'étude tout comme celle de décliner d'autres scénarios pour d'autres types de soins ou de traitements.

Le jeu sérieux pourrait également être utilisé par séquences ou dans son intégralité à différentes étapes des formations prégraduées des médecins et infirmières en collaboration avec les hautes écoles de santé et les universités de médecine.

\section{Conclusion}

Cette démarche interprofessionnelle de construction d'un jeu sérieux s'inscrit dans une démarche de formation continue multimodale. Des aménagements du dispositif sont nécessaires et envisagés afin d'optimiser l'impact de cette formation. Notre expérience de jeu sérieux dans la formation aux bonnes pratiques en matière de gestion de la douleur nous permet d'affirmer que cette modalité pédagogique est très prometteuse dans ce domaine.

Liens d'intérêts : Les auteurs déclarent ne pas avoir de liens d'intérêts.

\section{Références}

1. Bollondi C, Escher M, Boegli M, et al (2010) Why do nurses fail to rescue their patients? Communication in Congrès EAPC Glasgow

2. Davies AN (2011) The management of breakthrough cancer pain. Br J Nurse 20(13) :803-807doi: 10.12968/bjon.2011.20.13.803

3. Verloo H, Cohen C, BorlozC, et al (2013) Risks associated with the use of morphine for analgesia: attitudes and perceptions amongst nursing students in French-speaking Switzerland. Nursing 3:1-8. doi10.2147/NRR.S39401

4. Shaheen PE, Legrand SB, Walsh D, et al (2010) Errors in opioid prescribing: a prospective survey in cancer pain. J Pain Symptom Manage 39:702-11. doi: 10.1016/j.jpainsymman.2009.09.009

5. Murnion BP, Gnjidic D, Hilmer SN (2010) Prescription and administration of opioids to hospital in-patients, and barriers to effective use. Pain Med 11:58-66. doi: 10.1111/j.1526-4637.2009.00747.x

6. Organisation mondiale de la santé 2013. [Consulté le 12 novembre 2018]; http://www.who.int/gpsc/5may/5may2013_patient-participation/fr/

7. Touveneau S, Benichou A, Geissbuhler A, Merkli S (2018) Démarche relationnelle du partenariat entre patients et professionnels : conception et implémentation. Rev Med Suisse 14:1533-7

8. Sanchez E, Ney M, Labat JM (2011) Jeux sérieux et pédagogie universitaire : de la conception à l'évaluation des apprentissages. Rev Int Technol Pedagog Univ 8:48-57

9. Ingadottir B, Blondal K, Thue D, et al (2017) Development, usability and efficacy of a serious game to help patients learn about pain management after surgery: an evaluation study. JMIR Serious Games 5:e10. doi: 10.2196/games.6894

10. Creutzfeldt J, Hedman L, Fellander-Tsai L (2012) Effects of pretraining using serious game technology on CPR performance - an exploratory quasi-experimental transfer study. Scand J Trauma Resusc Emerg Med 20:79. doi: 10.1186/1757-7241-20-79

11. Boeker M, Andel P, Vach W, Frankenschmidt A (2013) Gamebased e-learning is more effective than a conventional instructional method: a randomized controlled trial with third-year medical students. PLoS One 8:e82328. doi: 10.1371/journal.pone.0082328

12. Dankbaar M (2017) Serious games and blended learning; effects on performance and motivation in medical education. Perspect Med Educ 6:58-60. doi: 10.1007/s40037-016-0320-2

13. Richard C, Lussier MT (2016) La communication professionnelle en santé. $2^{\mathrm{e}}$ édition, Pearson, Montréal 
14. Olszewski AE, Wolbrink TA (2017) Serious gaming in medical education: a proposed structured framework for game development. Simul Healthc 12:240-53. doi: 10.1097/SIH.0000000000000212

15. Graafland M, Schraagen JM, Schijven MP (2012) Systematic review of serious games for medical education and surgical skills training. Br J Surg 99:1322-30. doi: 10.1002/bjs.881

16. Wang R, Demaria S Jr, Golberg A, Katz D (2016) A systematic review of serious games in training health care professionnals. Simul Healthe 11:41-51. doi 10.1097/SIH000000000000118

17. Hamari J, Shernoff DJ, Rowe E, et al (2016) Challenging games help students learn: an empirical study on engagement, flow and immersion in game-based learning. Comput Human Behav 54:170-9. doi: org/10.1016/j.chb.2015.07.045

18. Drummond D, Hadchouel A, Tesnière A (2017) Serious games for health: three steps forwards. Adv Simul 2:3. doi 10.1186/ s41077-017-0036-3

19. Akl EA, Kairouz VF, Sackett, KM, et al (2013) Educational games for health professionals. Cochrane Database Syst Rev 3: CD006411. doi:10.1002/14651858.CD006411.pub4

20. Sipiyaruk K, Gallagher JE, Hatzipanagos S, Reynolds PA (2018) A rapid review of serious games: from healthcare education to dental education. Eur J Dent Educ 22:243-57. doi: 10.1111/eje.12338 